\title{
Clinico-morphological Profile of Cutaneous Fungal Infections: An Experience from a Tertiary Care Government Hospital in North India
}

\author{
Ekta Jain ${ }^{1 *}$, Rajpal Singh Punia ${ }^{2}$, Jagdish Chander $^{3}$ and Mala Bhalla ${ }^{4}$ \\ 'Core Diagnostics, 406, Udyog Vihar III, Gurgaon 122001 \\ ${ }^{2}$ Department of Pathology Government Medical College and Hospital, Sector 32, Chandigarh \\ ${ }^{3}$ Department of Microbiology, Government Medical College and Hospital, Sector 32, Chandigarh \\ ${ }^{4}$ Department of Dermatology and Venerology, Government Medical College and Hospital, Sector 32, Chandigarh
}

\begin{abstract}
Background: Cutaneousfungal infections are predominantly seen in hot tropical countries like India. In the past decade, there has been an escalation in recurrent and chronic fungal infections. Skin biopsy may play a critical role in rapid identification of these infections.

Methods: Fifteen cases of cutaneous fungal infections over a period of 6 years were included. Formalin-fixed tissue was subjected to hematoxylin and eosin and histochemical staining including Gomori Methanamine Silver and Periodic Acid Schiff. Results of KOH smear test and fungal culture were included wherever available. The clinico-morphological patterns in various cutaneous fungal infections was evaluated.

Results: Most patients were between 25 to 40 years of age and commonly presented as skin ulceration, followed by nodular swelling and multiple discharging sinuses. Candidiasis and Mycetoma infections were the commonest infections. Others included: Cryptococcosis, Dermatophytosis, Chromoblastomycosis and Mucormycosis. The predominant histopathologic patterns were perivascular and interstitial inflammation. Eleven cases were confirmed by $\mathrm{KOH}$ examination and culture.

Conclusion: The morphologic spectrum of cutaneous fungal infections is varied.Initial presentations of these fungal infections may be indicative of the onset of a life-threatening systemic mycoses. Thus, the histopathologic evaluation of skin tissue specimens is critical for their rapid and accurate diagnosis.
\end{abstract}

Keywords: Culture, Cutaneous Fungal Infections,Dermatophytes, Pas Staining, Skin Biopsy

\section{Introduction}

Fungal infections are recognized as causative agents of human diseases earlier than bacteria. Although fungi are world-wide, only few of them are considered pathogenic. [1] These common pathogens occur in varying degrees of severity, ranging from asymptomatic infections to fatal diseases. Various environmental, physiologic, socioeconomic and host factors can contribute to the development of fungal diseases. Low socioeconomic status, poor hygiene, overcrowding, improper sanitation, lack of health education and awareness, and poor health-care facilities are the most important predisposing parameters when combined with exposure to etiologic fungi. ${ }^{[2,3]}$

Localized colonization of the skin (primary infection) or inhalation of spores (secondary/systemic infection) may give rise to persistent infections; therefore, mycoses often begin on skin or in the lungs. ${ }^{[4]}$ Fungal infections involving the skin can be either superficial or subcutaneous mycosis. ${ }^{[5]}$ The prevalence of superficial mycotic infection worldwide according to the World Health Organization has been found to be $20 \%-25 \%{ }^{[6]}$ These infections are predominantly seen in hot tropical countries like India. In the past decade, there has been an escalation in difficult to treat, recurrent as well as chronic fungal infections in India. ${ }^{[5,7,8]}$ This increase may be a result of frequent usage of antibiotics, immunosuppressive drugs and various conditions like organ transplantations, lymphomas, leukemia and human immunodeficiency virus (HIV) infections. ${ }^{[9,10,11]}$ Rapid and accurate diagnosis of cutaneous fungal infections is essential for early, appropriate treatment as initial presentations in the skin may be indicative underlying immunodeficiency and onset of a life-threatening systemic mycoses.

Many diagnostic modalities are available to diagnose these pathogenic fungal infections such as $\mathrm{KOH}$ smear and culture, histopathological examination, serology, histochemical stains like Gomori methenamine silver (GMS), Periodic acid-Schiff (PAS), immunoperoxidase stains and polymerase chain reaction (PCR) etc. Culture is considered to be the Gold Standard, but it has a variable and longer turnaround time. Thus, routine histopathologic examination of the lesional skin remains the primary method of confirming a cutaneous fungal infection because it permits fast, presumptive identification of fungi. 


\section{Materials and Methods}

This retrospective study was done over a period of 6 years at a tertiary care government hospital in north India. Fifteen cases diagnosed as cutaneous fungal infections were included in the study. All patients having superficial or deep fungal infections including Diabetic/ immunocompromised patients and those who had not been treated earlier for the fungal infection were included in the study. Any patient with a known history of topical or systemic antifungal treatment was excluded from the study.

In all fifteen cases, from formalin-fixed paraffin-embedded tissues, three to five-micrometer-thick sections were obtained and subjected to hematoxylin and eosin (H\&E) staining and histochemical stains including Gomori Methanamine Silver (GMS) and Periodic Acid Schiff (PAS) using standard protocols. ${ }^{[12]}$ The clinical data was retrieved from archival records. Results of $\mathrm{KOH}$ test and fungal culture were included wherever available. The clinicomorphological profile in all the cases was evaluated.

As anonymized patient reports were used, ethical permission was not required for the study.

\section{Results}

In the study, age of the patients ranged from 20 to 71 years with most patients between 25 to 40 years. Out of 15 patients, $10(66.67 \%)$ were males and $5(33.3 \%)$ were females. Male to female ratio was 2:1. Areas of involvement included groins (4/15), lower extremities $(3 / 15)$, trunk (1/15), multiple sites of skin involvement $(3 / 15)$, gluteal region $(2 / 15)$ and scalp (1/15). The most common clinical presentation was skin ulceration $(\mathrm{n}=7,47 \%)$ followed by nodular swelling $(\mathrm{n}=4$, $27 \%)$. Multiple discharging sinuses $(n=2,13 \%)$ and hyperpigmented lesions $(n=2,13 \%)$ were the other presentations. Candidiasis and Mycetoma infections were the commonest diagnosed fungal infections. Candida was seen as pseudohyphae and budding yeast forms. Mycetoma was identified by radially arranged, filamentous septate, branched hyphae with round to oval cells at the periphery. Encapsulated yeast-like fungus with numerous spores surrounded by a halo led to diagnosis of Cryptococcus. Fungal arthrospores and hyphae in stratum corneum were diagnosed as Dermatophytes (Tinea barbae and Tinea Cutis). One case revealed dematiaceous fungi comprising pigmented, thick walled, ovoid spores varying in size from 6-12 $\mu$, lying in clusters and were identified as Chromoblastomycosis. Mucormycosis was seen as broad, aseptate, right angled branched fungal hyphae. Distribution of various fungal infections is shown in Table 1. Around 3 cases of the fungal infections could not be accurately classified as culture studies were not available for the same.

Various histopathological features and fungal profiles seen on H\&E sections as well as PAS stains are shown in Tables 2 and 3 and Figures 1-4.

In the present study, all the cases were due to primary infection. No case was secondary to a systemic disease. Out of 15 cases, histochemical stains (PAS, GMS) highlighted the fungal profiles in 12 cases. Eleven out of 15 cases were confirmed by $\mathrm{KOH}$ microscopic examination and culture. In 4 cases, $\mathrm{KOH}$ test and culture results could not be retrieved.

Table 1: Distribution of fungal infections.

\begin{tabular}{|l|l|}
\hline Fungal infection & No. of cases (\%) \\
\hline Candida & $3(20)$ \\
\hline Mycetoma & $3(20)$ \\
\hline Cryptococcus & $2(13)$ \\
\hline Dermatophytes & $2(13)$ \\
\hline Chromoblastomycosis & $1(7)$ \\
\hline Mucor & $1(7)$ \\
\hline Unclassifiable & $3(20)$ \\
\hline
\end{tabular}

Table 2: Histopathological features on $\mathrm{H}$ and E staining.

\begin{tabular}{|l|l|l|}
\hline S.No & Histopathological features & No. of cases (\%) \\
\hline 1. & Epidermal changes & \\
\hline & Pseudoepitheliomatous hyperplasia & $5(33.3 \%)$ \\
\hline & Atrophy & $2(13.3 \%)$ \\
\hline & Acanthosis & $8(53.3 \%)$ \\
\hline & Hyperkeratosis & $6(40 \%)$ \\
\hline & Parakeratosis & $3(20 \%)$ \\
\hline & Spongiosis & $4(26.7 \%)$ \\
\hline
\end{tabular}




\begin{tabular}{|l|l|l|}
\hline S.No & Histopathological features & No. of cases (\%) \\
\hline & Surface ulceration & $4(26.7 \%)$ \\
\hline & Neutrophilic exocytosis & $2(13.3 \%)$ \\
\hline 2. & Dermal changes & $\begin{array}{l}\text { Upper and mid }-4(26.7 \%), \\
\text { Pandermal - 6(40\%) }\end{array}$ \\
\hline & Inflammatory infiltrate & $6(40 \%)$ \\
\hline & Histiocytic granulomas & $5(33.3 \%)$ \\
\hline & Giant cells & $5(33.3 \%)$ \\
\hline & Neutrophilic abscesses & $4(26.7 \%)$ \\
\hline & Necrosis & $1(13.3 \%)$ \\
\hline & Epithelioid cell granulomas & $2(26.7 \%)$ \\
\hline & Pigment incontinence & \\
\hline
\end{tabular}

Table 3: Pattern of various fungal profiles.

\begin{tabular}{|l|l|l|}
\hline S.No & Fungal morphology & No. of cases (\%) \\
\hline 1. & Location & $11(73.3 \%)$ \\
\hline & Dermis & Stratum corneum $-3(20 \%)$, Keratin layer $-1(13.3 \%)$ \\
\hline & Epidermis & $3(20 \%)$ \\
\hline 2. & Giant cells & \\
\hline & Fungal profile: & $3(20 \%)$ \\
\hline & Spores & $\begin{array}{l}\text { Septate-3 }(20 \%), \\
\text { Aseptate-1 }(13.3 \%)\end{array}$ \\
\hline & True hyphae & $3(20 \%)$ \\
\hline & Pseudohyphae & $5(33.3 \%)$ \\
\hline & Yeast forms & $3(20 \%)$ \\
\hline
\end{tabular}

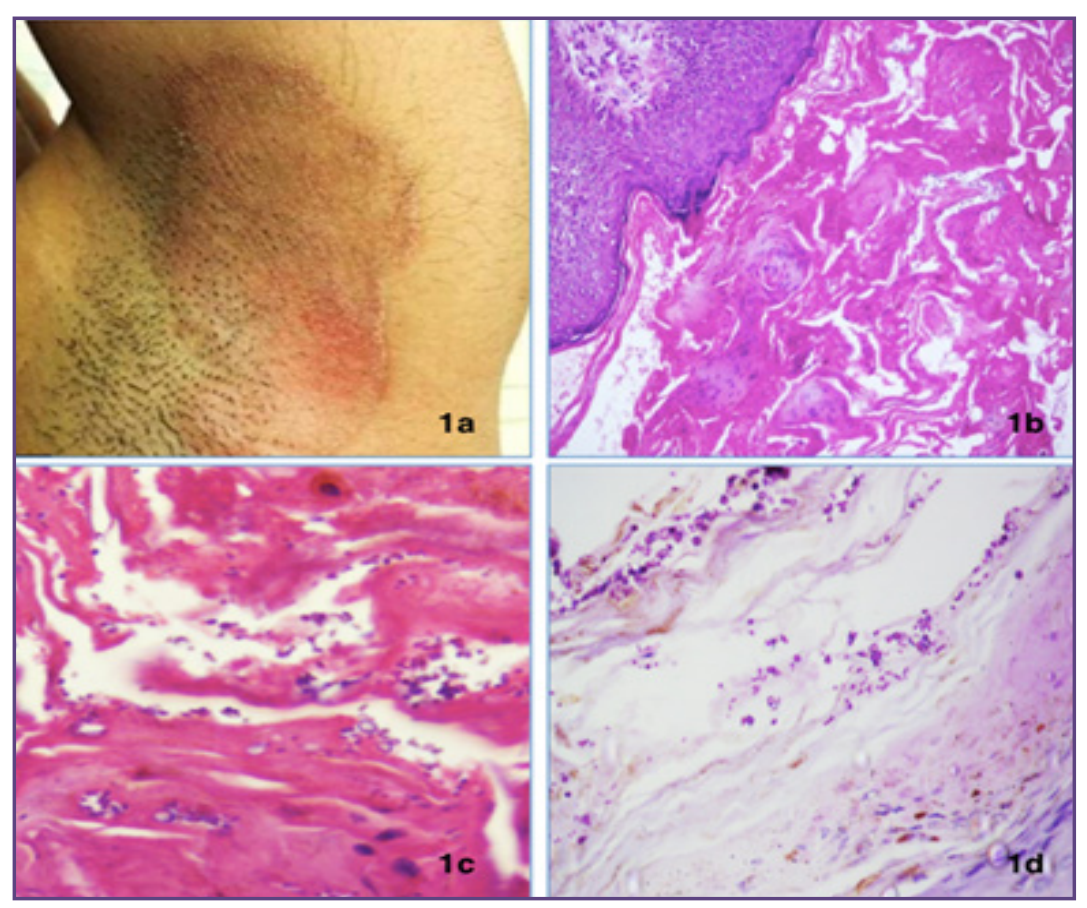

Fig. 1. 1a. Tinea Corporis infection presenting as well-defined margins and diffuse erythema, $\mathrm{H}$ and $\mathrm{E}$ section showing epidermal hyperkeratosis and parakeratosis admixed with arthrospores of Tinea Corporis. (H\&Ex100) (Fig 1b), High power view of the spores of Tinea Corporis (H\&Ex200) (Fig 1c), Arthrospores of Tinea Corporis showing good PAS positivity (PASx200) (Fig 1d). 


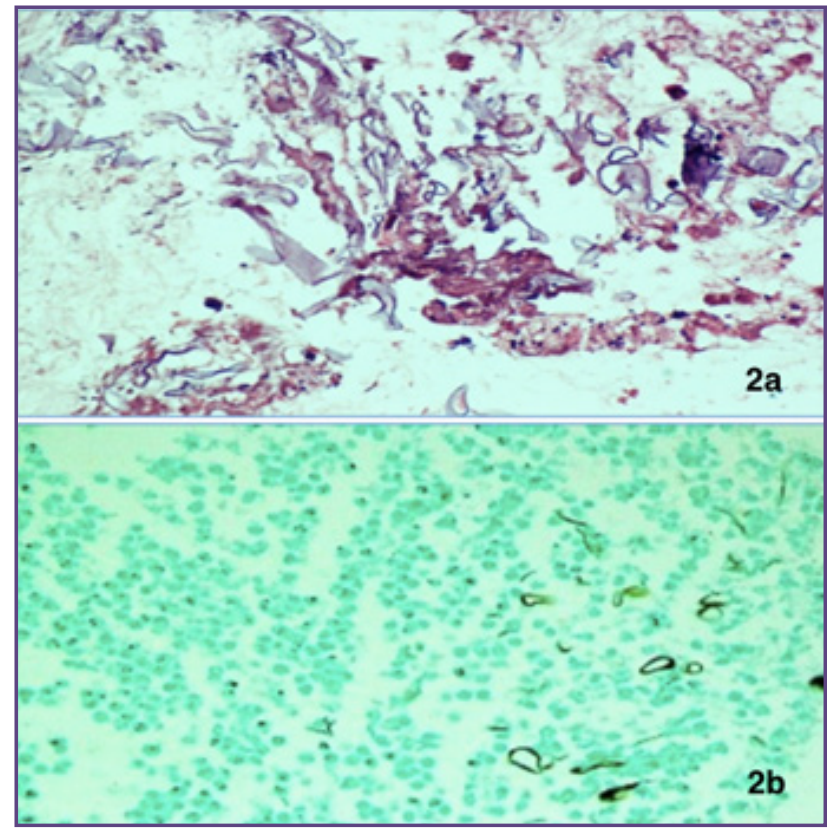

Fig. 2. 2a. Mucormycosis, $\mathrm{H}$ and E section showing broad, aseptate, right angle branched fungal hyphae (H\&Ex600), highlighted on GMS (GMSx200) (Fig 2b).

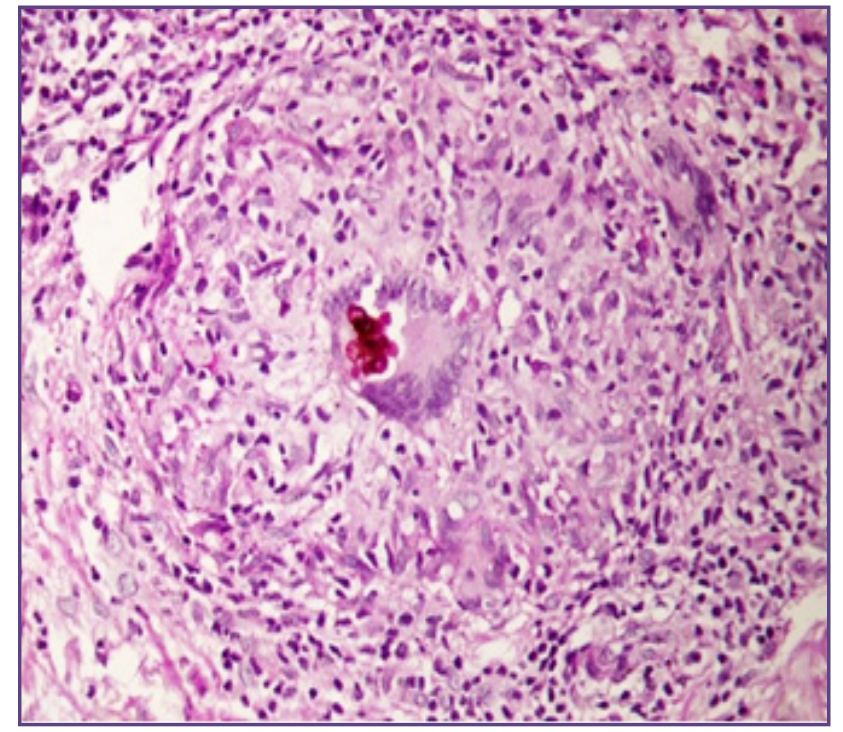

Fig. 3. Chromoblastomycosis. Low power view showing epithelioid cells with multinucleated giant cells in dermis. One giant cell shows presence of pigmented, thick walled, ovoid spores varying in size from 6-12 $\mu$ and lying in clusters (H\&Ex200).

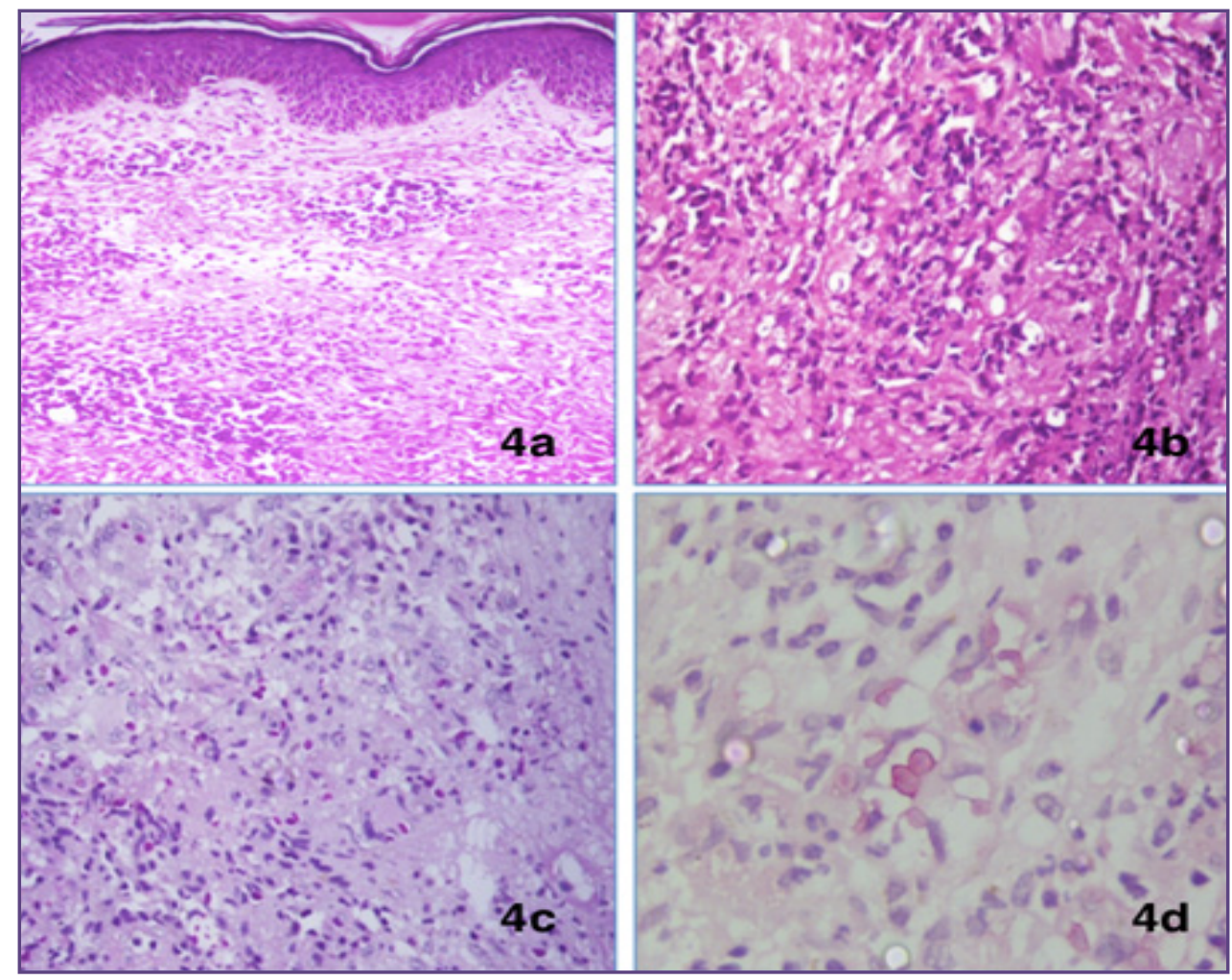

Fig. 4. 4a. Cryptococcosis, Low power view showing numerous spores of cryptococcus fungus surrounded by dense lymphoplasmacytic cell infiltrate and epithelioid cell granulomas (H\&Ex200), high power view showing the spores of cryptococcus having halo (H\&Ex400) (Fig 4b), spores highlighted by PAS staining (PASx400) (Fig 4c) while capsule highlighted by Mucicarmine staining (Mucicarminex1000) (Fig 4d). 


\section{Discussion}

Superficial and cutaneous fungal infections are among the most widespread groups of mycoses caused by a number of pathogens. Mycoses are classified according to the tissue levels initially colonized into primary and secondary (systemic) infections. ${ }^{[4]}$

Primary infections can be divided into two groups: those causing superficial infections and those that cause deep infections.

- Superficial dermatomycoses: These are limited to the outermost layers of the skin and hair. Various organisms include dermatophytes, Candida species, Malassezia furfur, Cladosporium, etc.

- Deep dermatomycoses: These involve the dermis, subcutaneous tissues, muscle and fascia. These include aspergillosis, chromomycosis, phaeohyphomycosis, eumycetoma, rhinosporidiosis and lobomycosis.

Clinical manifestations vary depending on the causative agent and on the host immune response. Histopathological diagnosis is based on finding of specific fungal profile. The histopathologic fungal detection with PAS staining possesses high sensitivity, thus skin biopsies may be critical for their early diagnosis.

In our study, out of 15 cases, 8 were causing superficial mycoses while 7 were causing deep mycoses. The prevalence of cutaneous fungal infections was higher in males $(66.67 \%)$ which was similar to another study wherein these infections were more common in males $(50.9 \%)$ in comparison to females. ${ }^{[13]}$ On the other hand, another study indicated higher incidence of cutaneous fungal infections in females. ${ }^{[14]}$ The commonest age group was $25-40$ years which was similar to other studies. ${ }^{[7,15]}$ This can be explained as this is the age group of maximum outdoor activities, thus more exposure to heat and humidity which is an important predisposing factor for fungal infections. The most common clinical presentation in our study was skin ulceration, nodules and sinus formation while another study showed more annular plaques, papules and pustules. ${ }^{[5]}$

The most common area of involvement was groins $(4 / 15$, $26.7 \%)$ followed by lower extremities $(3 / 15,20 \%)$, gluteal region $(2 / 15,13.3 \%)$, trunk $(1 / 15,6.7 \%)$ and scalp $(1 / 15$, $6.7 \%)$. Multiple sites of infection were found in $3 / 15(20 \%)$ cases. These findings were similar to another study where groin was the commonest area involved (19\%), followed by proximal part of leg and arm (15\%), finger and toe nail infection $(12 \%)$, trunk $(12 \%)$, and less affected area was scalp $(1 \%)^{[3]}$ while multiple sites of involvement has been reported around $17-30 \%$ in previous studies. ${ }^{[15]}$ Thus, fungal infections are more common in areas more prone to sweating and sweat retention that provides a conducive environment for fungal growth.

Dermatophytes have always been among the commonest microorganisms causing superficial mycoses in many tropical countries including India. ${ }^{[16]}$ Our study showed an increase in the incidence of non-dermatophytic infections (87\%) most common being candidiasis (20\%) and mycetoma $(20 \%)$ infections. The prevalence of Dermatomycosis was $13 \%$ in the present study compared to $75.6 \%$ in a previously reported study by Lakshmanan et a ${ }^{[7]}$ which also showed increase in numbers of non- dermatophytic infections with candida being the commonest infection (14.6\%).

The predominant histopathologic patterns in our study were perivascular and interstitial inflammation (10/15), epidermal acanthosis $(8 / 15)$, granulomatous inflammation (7/15), hyperkeratosis (6/15), pseudoepitheliomatous hyperplasia (5/15), neutrophilic abscesses (5/15) and necrosis $(4 / 15)$. This was in comparison to other study where granulomatous inflammation (18/33) was most common followed by pseudoepitheliomatous hyperplasia $(2 / 33)$, perivascular and interstitial inflammation without granulomas (1/33), small vessel vasculitis (1/33), and necrosis $(1 / 33) .^{[17]}$ This shows that the basic pattern of cutaneous infection is relatively uniform. The degree of inflammatory response, however, varies based on a number of factors, particularly on host immune response.

Histochemical stains (PAS, GMS) highlighted the fungal profiles in 12 cases. Eleven out of 15 cases were confirmed by $\mathrm{KOH}$ microscopic examination and culture studies. This reflects relatively high diagnostic accuracy of histopathology for cutaneous fungal infections in our practice. These concordance rates are similar to previously reported studies on culture and pathology correlations showing that overall diagnostic accuracy for microscopic morphologic techniques ranges from $20 \%$ to $80 \%$. ${ }^{[18,19]}$

Even though the morphologic features of various fungal organisms may overlap, the histopathologic examination remains one of the major diagnostic modalities because it permits a rapid, presumptive identification of fungal infections. Various pitfalls in identifying fungal organisms include: 1) An accurate assessment of septation and type of branching may not be possible when fungal hyphae are scanty, folded, kinked, or fragmented, 2) Presence of extensive necrosis may lead to a more swollen and distorted appearance of hyphae and thus an erroneous diagnosis, 3) Antifungal medications and host responses may lead to alteration of fungal characteristics, 4) Tissue processing and inflammatory processes may lead to fragmentation of fungal elements, thus obscuring fungal morphology. ${ }^{[20]}$ 
In order to avoid misidentification of various fungi, the close differential diagnosis need to be excluded. Dermatophytes, which appear as hyaline septate fungi should be differentiated from Aspergillus that share similar morphologic appearance showing branched, uniform, septate hyphae. Similarly, histologic differentiation of Candida from other smaller yeast like Histoplasma can be challenging. Although typically present extracellularly, intracellular Candida can mimic Histoplasma. Similarly, extracellular Histoplasma may show overlapping findings with Candida. The unique small size and lack of pseudohyphae production of Candida can further obscure accurate identification. Pointers towards identification of Candida include variably sized yeast cells that produces pseudohyphae with lack of a pseudocapsule. Also, Candida typically leads to a suppurative tissue reaction, whereas Histoplasma elicits a more granulomatous reaction. Candida also has to be differentiated from other yeasts that produce true hyphae in tissue. The most frequent differential diagnosis is with Aspergillus and Trichosporon. The pseudohyphae of Candida appear to be branching but are differentiated because these are slender and aseptate.

Cryptococcosis may sometimes produce lesser amount of polysaccharide capsule; thus, may resemble other yeasts of similar size, such as Candida or Histoplasma. Staining these specimens with Masson-Fontana stain may prove that the yeast produces melanin, which is characteristic of cryptococci. The major morphological differentiation between Mucor genera is with other fungi that produce non-pigmented hyphae in tissue, including Aspergillus, other hyaline septated molds (such as Fusarium), and Candida. The presence of abundant septation and acuteangle branching should suggest the diagnosis of Aspergillus while yeasts with pseudohyphae should suggest Candida. Poor staining of hyphae with GMS should suggest mucormycosis. The different dematiaceous fungi cannot be distinguished from one another by histology. Some dematiaceous fungi may show very little melanin and may appear as hyaline hyphae; thus, a Masson Fontana stain is needed to highlight the pigment. In all such cases, there is a potential for misdiagnosis and careful evaluation of fungal profiles is essential to arrive at accurate diagnosis and avoid inappropriate pharmacotherapy. ${ }^{[21]}$

The clinical diagnosis of a cutaneous fungal infection can be challenging. In most cases, while an infection may be suspected, a specific infectious agent cannot be implicated solely on the basis of the clinical findings. Skin lesions are nonspecific, requiring adjunctive pathologic examination and skin tissue cultures for diagnostic confirmation.

The limitations of our study included the fact that this was a retrospective study of small number of cases $(n=15)$ without any follow-up details of the patients from a single tertiary care institution. There is paucity of literature on skin changes in cutaneous fungal infections. Thus, future studies with more number of cases with adequate information on follow-up may help in identifying cutaneous changes in fungal infections to initiate appropriate therapy.

\section{Conclusion}

The morphologic spectrum of cutaneous fungal infections is varied. Skin is a common site for colonization by fungal pathogens. The initial presentations of fungal infections in the skin may be indicative of the onset of a life-threatening systemic mycoses. Thus, the histopathologic evaluation of skin tissue specimens is critical for rapid and accurate diagnosis of cutaneous fungal infections.

\section{References}

1. Kannan P, Janaki C and Selvi GS (2006): Prevalence of dermatophytes and other fungal agents isolated from clinical samples. Indian J Med Microbiol., 24(3): 212-215.

2. Oke OO, Onayemi O, Olasode OA, Omisore AG, Oninla $\mathrm{OA}$. The prevalence and pattern of superficial fungal infections among school children in ile-ife, South-Western Nigeria. Dermatol Res Pract 2014.

3. Narasimhalu CR, Kalyani M, Somendar S. A cross-sectional, clinico-mycological research study of prevalence, aetiology, speciation and sensitivity of superficial fungal infection in Indian patients. J Clin Exp Dermatol Res. 2016;7(324):2.

4. Molly H, Longley J. Fungal diseases. In: Lever's histopathology of the skin. Lipincott Williams and Wilkins. 9th ed. 2005;591-620.

5. Dash M, Panda M, Patro N, Mohapatra M. Sociodemographic profile and pattern of superficial dermatophytic infections among pediatric population in a tertiary care teaching hospital in Odisha. Indian Journal of Paediatric Dermatology. 2017;18(3):191-5.

6. WHO, 2005. Epidemiology and management of common skin diseases in children in developing countries. World Health Organization, Geneva. WHO/FCH/CAH/05.12.

7. Lakshmanan A, Ganeshkumar P, Mohan SR, Hemamalini M, Madhavan R. Epidemiological and clinical pattern of dermatomycoses in rural India. Indian journal of medical microbiology. 2015;33(5):134-6.

8. Shenoy MM, Jayaraman J. Epidemic of difficult-to-treat tinea in India: Current scenario, culprits, and curbing strategies. Arch Med Health Sci 2019;7:112-7.

9. Petmy LJ, Lando AJ, Kaptue L, Tchinda V, Folefack M. Superficial mycoses and HIV infection in Yaounde. J Eur Acad Deramtol Venereol 2004; 8: 301-4

10. Kim MS, Lee SM, Sung HS, Won CH, Chang S, Lee MW, et al. Clinical analysis of deep cutaneous mycoses: a 12-year experience at a single institution. Mycoses 2012;55:501-6.

11. Singh N. Fungal infection in the recipients of solid organ transplantation. Infect Dis Clin North Am 2003;17:113. 
12. Jackson $\mathrm{P}$, Blythe D. Immunohistochemical techniques. In: Bancroft JD, Gamble M, editors. Theory and practice of histological techniques. 6th ed. Philadelphia: Elsevier/ Churchill Livingstone; 2008. 433-69.

13. Berenji F, Sivaki MM, Sadabadi F, Aliabadi ZA, Ganjbakhsh M, Salehi M. A retrospective study of cutaneous fungal infections in patients referred to Imam Reza Hospital of Mashhad, Iran during 2000-2011. Current medical mycology. 2016;2(1):20.

14. Miklić P, Skerlev M, Budimčić D, Lipozenčić J. The frequency of superficial mycoses according to agents isolated during a ten-year period (1999- 2008) in Zagreb area, Croatia. Acta Dermatovenerol Croat. 2010;18(2):92-8.

15. Grover S, Roy P. Clinico-mycological profile of superficial mycosis in a hospital in North-East India. Medical journal armed forces India. 2003;59(2):114-6.

16. Mandell, Dauglus Bennett's. Principle and Practice of Infectious Diseases, Sixth Edition, Volume 2, Chapter 265, Dermatophytosis and Other Superficial Mycoses :3051-62.
17. Santiago TM, Pritt B, Gibson LE, Comfere NI. Diagnosis of deep cutaneous fungal infections: Correlation between skin tissue culture and histopathology. Journal of the American Academy of Dermatology. 2014;71(2):293-301.

18. Schofield CM, Murray CK, Horvath EE, Cancio LC, Kim $\mathrm{SH}$, Wolf SE, et al. Correlation of culture with histopathology in fungal burn wound colonization and infection. Burns 2007;33:341-6.

19. Guarner J, Brandt M. Histopathologic diagnosis of fungal infections in the 21st century. Clin Microbiol Rev 2011;24: 247-80.

20. Sangoi AR, Rogers WM, Longacre TA, Montoya JG, Baron EJ, Banaei N. Challenges and pitfalls of morphologic identification of fungal infections in histologic and cytologic specimens: a ten-year retrospective review at a single institution. American journal of clinical pathology. 2009 Mar 1;131(3):364-75.

21. Guarner J, Brandt ME. Histopathologic diagnosis of fungal infections in the 21 st century. Clinical microbiology reviews. 2011 Apr 1;24(2):247-80.

*Corresponding author:

Dr. Ekta Jain, MD, DNB, Pathologist- Senior Associate, Core Diagnostics, 406, Udyog Vihar III, Gurgaon 122001

Phone: +91 8054777455

Email: ektajain86@gmail.com

Date of Submission $\quad: 17 / 10 / 2020$

Date of Final Revision : 22/01/2021

Financial or other Competing Interests: None.

Date of Acceptance $\quad: 26 / 01 / 2021$

Date of Publication $\quad: 28 / 02 / 2021$ 